\title{
Observation and Correction of Resonance Stopbands in the AGS Booster*
}

\author{
C. Gardner, Y. Shojił L. Ahrens, J.W. Glenn, Y.Y. Lee, T. Roser \\ A. Soukas, W. van Asselt, and W.T. Weng \\ AGS Department, Brookhaven National Laboratory \\ Upton, New York 11973
}

REC
JUW 281993

\section{Abstract}

At the design intensity of $1.5 \times 10^{13} \mathrm{ppp}$, the space charge tune shift in the AGS Booster at injection has been estimated to be about 0.35 . Therefore, the beam is spread over many lower order resonance lines and the stopbands have to be corrected to minimize the amplitude growth by proper compensation of the driving harmonics resulting from random errors. The observation and correction of second and third order resonance stopbands in the AGS Booster, and the establishment of a favorable operating point at high intensity are discussed.

\section{INTRODUCTION}

The AGS Booster began operation [1] in 1992, delivering beam at one third the design intensity of $1.5 \times 10^{13} \mathrm{ppp}$. An important part of the ongoing effort to reach the design intensity has been the correction of the second and third order resonance lines encountered by the beam during injection and early acceleration. The Booster operates with the horizontal and vertical tunes $\left(Q_{x}\right.$ and $\left.Q_{y}\right)$ between four and five, and at the design intensity the space charge tune shift has been estimated to be about 0.35 at injection. With the nominal operating point at $Q_{x}=4.82$, $Q_{y}=4.83$ several of the second and third order lines shown in Figure 1 are therefore encountered and the associated stopbands must be corrected in order to minimize beam loss. The second and third order resonances and the types of field which excite them are listed below:

Second Order (excited by quadrupole fields):

$$
2 Q_{x}=9,2 Q_{y}=9
$$

Second Order (excited by skew quadrupole fields):

$$
Q_{x}+Q_{y}=9, Q_{x}-Q_{y}=0
$$

Third Order (excited by sextupole fields):

$$
\begin{aligned}
& 3 Q_{x}=14, Q_{x}+2 Q_{y}=14 \\
& 3 Q_{x}=13, Q_{x}+2 Q_{y}=13
\end{aligned}
$$

"Work performed under the auspices of the U.S. Dept. of Energy.

tPermanent address, KEK

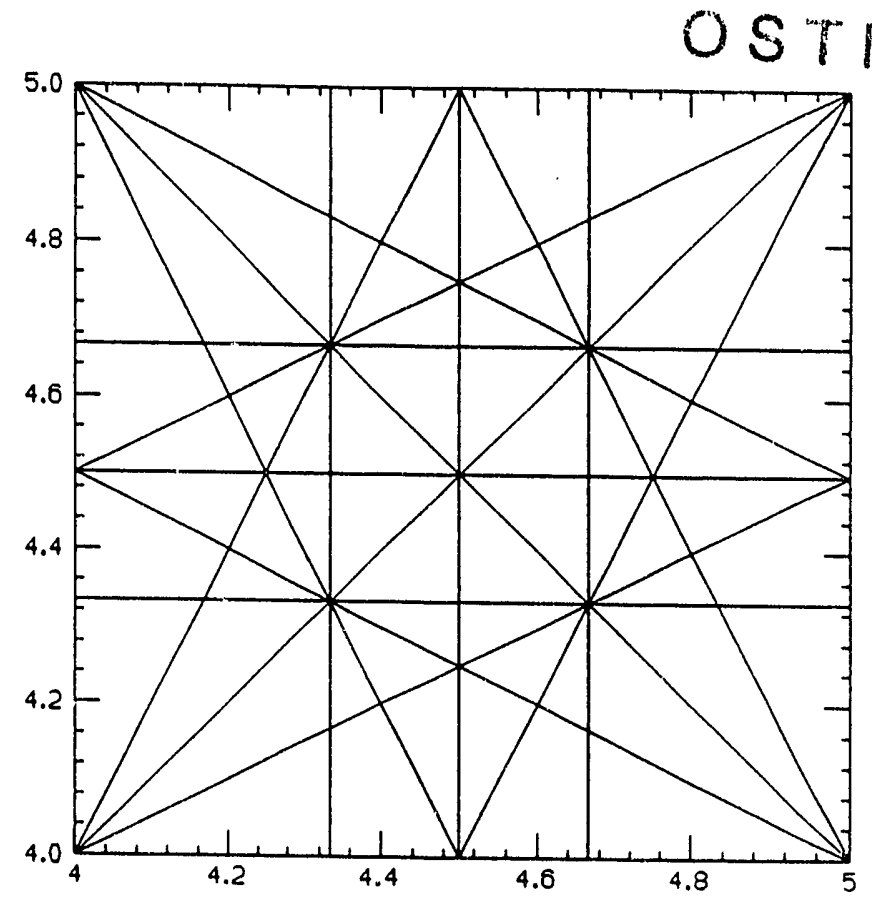

Figure 1: 2nd and 3rd Order Resonances

$$
2 Q_{y}-Q_{x}=5,2 Q_{y}-Q_{x}=4
$$

Third Order (excited by skew sextupole fields):

$$
\begin{gathered}
3 Q_{y}=14, Q_{y}+2 Q_{x}=14 \\
3 Q_{y}=13, Q_{y}+2 Q_{x}=13 \\
2 Q_{x}-Q_{y}=5,2 Q_{x}-Q_{y}=4
\end{gathered}
$$

The resonance correction system $[2,3]$ employs auxiliary windings placed on quadrupoles and sextupoles whose main windings serve to adjust the machine tunes and chromaticities. Special windings on the correction dipoles produce the required skew quadrupoles, and passive windings on the dipole vacuum chambers compensate the sextupole fields produced by eddy currents [4]. The various windings are excited so that appropriate azimuthal harmonics are produced which compensate the resonance driving harmonics resulting from random magnetic field errors. The system is capable of correcting resonances (1-4) simultaneously, i.e. it can correct each of these resonances without 


\section{DISCLAIMER}

This report was prepared as an account of work sponsored by an agency of the United States Government. Neither the United States Government nor any agency thereof, nor any of their employees, makes any warranty, express or implied, or assumes any legal liability or responsibility for the accuracy, completeness, or usefulness of any information, apparatus, product, or process disclosed, or represents that its use would not infringe privately owned rights. Reference herein to any specific commercial product, process, or service by trade name, trademark, manufacturer, or otherwise does not necessarily constitute or imply its endorsement, recommendation, or favoring by the United States Government or any agency thereof. The views and opinions of authors expressed herein do not necessarily state or reflect those of the United States Government or any agency thereof. 
affecting the correction of the others. The remaining resonances (5-8) are either difference resonances or resonances excited by skew sextupole fields. These were not considered in the development of the correction scheme because they were thought to be relatively weak. However, during the course of our studies we found that the skew sextupole resonances (6) are actually quite strong, and as a result, windings were placed on existing magnets in the ring to produce four skew sextupoles. Subsequent excitation of these magnets reduced losses significantly. Another unexpected source of beam loss was the presence of a strong ninth harmonic in the sextupole fields around the machine which, due to radial displacement of the orbit in the sextupoles, drives the quadrupole resonances (1). The loss was reduced by exciting available sextupole windings to produce a ninth harmonic sextupole field.

Resonances (1-3) and (6) were observed by programming the tunes to pass through each resonance at various times during the magnetic cycle. The beam intensity was reduced to ensure that the area occupied by the beam in tune space was as small as possible, and the loss upon traversal of each resonance was measured by observing a circulating beam current transformer. Figure 2 shows a typical trace of the beam current as a resonance is crossed. Here the upper and lower traces are respectively the beam current transformer and the current in the tune quadrupoles. The amount of loss was measured for several different correction settings in order to accurately determine the setting required to minimize the loss. These measurements were made at several different values of the field, $B$, and $d B / d t$ to determine the dependence of the required correction on these parameters. The results of the measurements and the effects of the corrections on the overall beam intensity are summarized in the following sections.

\section{QUADRUPOLE CORRECTION}

The corrections required for resonances (1) were found to have the following dependence on $B$ and $d B / d t$ :

$$
C_{x}=25+110 B+6.0 \dot{B}, S_{x}=-172+160 B-1.9 \dot{B}
$$

for the $2 Q_{x}=9$ resonance, and

$$
C_{y}=147+90 B+5.0 \dot{B}, S_{y}=-192+54 B-6.0 \dot{B}
$$

for the $2 Q_{y}=9$ resonance. Here $C$ and $S$ are proportional to the cosine and sine components of the 9th harmonic quadrupole field and the units of $B$ and $d B / d t$ are $\mathrm{kG}$ and $\mathrm{kG} / \mathrm{s}$. The units of correction have been chosen for tuning convenience.

During the course of determining these corrections we found that we could not completely iiminate the loss as the resonances were crossed, and with a crossing speed of $0.01 / \mathrm{ms}$ the residual loss was about $20 \%$. We initially thought that an 18th harmonic octupole field might be responsible for the loss, but then found that the required corrections vary linearly with the orbit radius which strongly

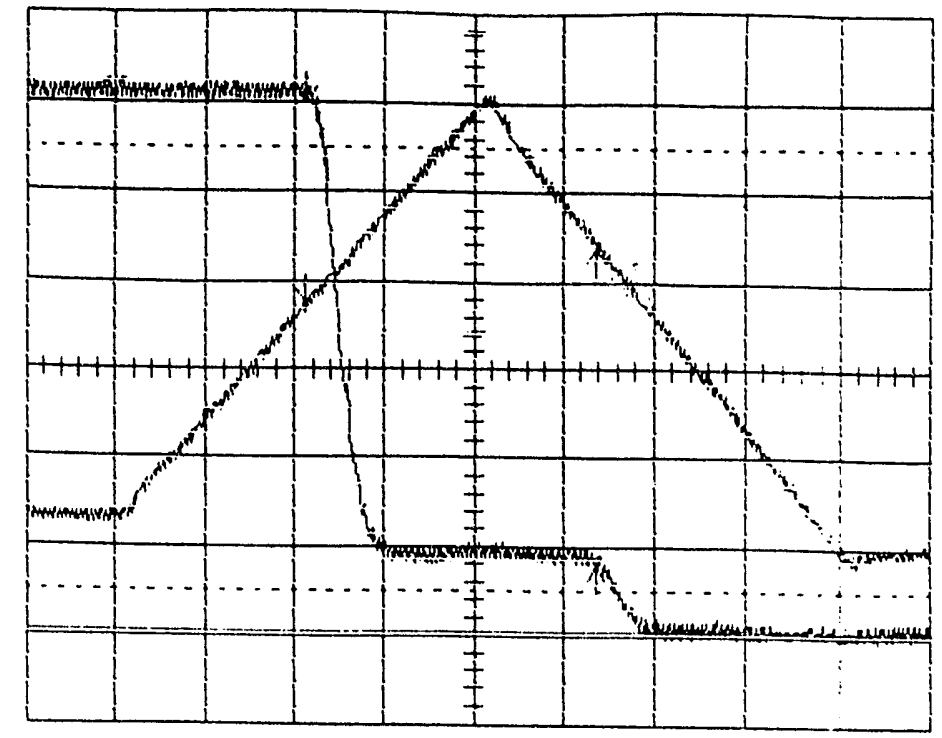

Figure 2: Beam Loss upon Crossing a Resonance.

suggested a ninth harmonic sextupole field. Beam particles whose momentum differs by $\delta p$ from the central momentum would then see a ninth harmonic quadrupole field which is proportional to $\delta p$ and this could produce the observed loss. We found that by introducing a ninth harmonic with available sextupole windings we could eliminate the dependence of the required correction on radius and raduce the residual loss to about $4 \%$. It is possible that the remanent fields of the sextupole magnets used to adjust the chromaticities are responsible for the ninth harmonic sextupole field.

\section{SKEW QUAD CORRECTION}

The corrections required for resonanies (2) were found to depend on $B$ but not on $d B / d t$ :

$$
C_{x y}=40+50 B, S_{x y}=-100+30 B
$$

for $Q_{x}+Q_{y}=9$ resonance, and

$$
C=-180+140 B
$$

for the $Q_{x}-Q_{y}=0$ resonance. Here $C_{x y}$ and $S_{x y}$ are proportional to the cosine and sine components of the 9th harmonic skew quadrupole fields and $C$ is proportional to the zeroth harmonic of these fields. As before, the units of correction have been chosen for tuning convenience.

As with the corrections for resonances (1) we found that we could not completely eliminate the loss as the $Q_{x}+$ $Q_{y}=9$ resonance was crossed, and with a crossing speed of $0.01 / \mathrm{ms}$ the residual loss was about $20 \%$. We suspect that the residual loss is due to a ninth harmonic skew sextupole field, but we have not yet confirmed this hypothesis. 


\section{SEXTUPOLE CORRECTION}

The corrections required for resonances (3) were found to depend on $d B / d t$ but only weatly on $B$ :

$$
C_{x}=30+0 B+3.5 \dot{B}, S_{x}=-90+20 B+6.0 \dot{B}
$$

for the $3 Q_{x}=14$ resonance, and

$$
C_{x y}=0+13 B+4.7 \dot{B}, S_{x y}=120+9 B+2.6 \dot{B}
$$

for the $Q_{x}+2 Q_{y}=14$ resonance. Here $C$ and $S$ are proportional to the cosine and sine components of the 14th harmonic sextupole fields.

\section{SKEW SEXT CORRECTON}

As previously mentioned, resonances (6) were found to be quite strong and as a result, additional windings were placed on existing magnets to produce four skew sextupoles. These were excited to produce the required 14th harmonic correction which was found to depend on $B$ and $d B / d t$ :

$$
C_{x y}=35+0 B+0 \dot{B}, S_{x y}=40-7.0 B+0.3 \dot{B}
$$

for the $Q_{y}+2 Q_{x}=14$ resonance. Here $C$ and $S$ are proportional to the cosine and sine components of the 14th harmonic skew sextupole fields. With this correction the loss upon traversal of the resonances at a crossing speed of $0.01 / \mathrm{ms}$ was reduced from $20 \%$ to $4 \%$.

\section{INTENSITY GAINS}

To see the effect of the corrections at high intensity, the corrections were programmed on the high intensity machine cycle according to their measured dependencies on $B$ and $d B / d t$. On this cycle beam is injected at $B=0.15 \mathrm{~T}$ with $d B / d t=3 \mathrm{~T} / \mathrm{s}$. Shortly after injection, $d B / d t$ increases to $7 \mathrm{~T} / \mathrm{s}$ and then decreases to $1 \mathrm{~T} / \mathrm{s}$ near extraction where $B=0.52 T$. The effect of the skew sextupole corrections is shown in Figure 3 where the upper and lower traces show the beam current with these corrections turned $O N$ and OFF. Here the peak intensity at injection was $14 \times 10^{12}$ and with the operating point of $Q_{x}=4.78, Q_{y}=4.94$ the final intensity just before extraction with the skew sextupole corrections turned $O N$ was $9.6 \times 10^{12}$. With these corrections turned OFF the final intensity decreased to $7.6 \times 10^{12}$ ppp. Turning off the sextupole corrections for resonances (3) further reduced the intensity by $0.7 \times 10^{12} \mathrm{ppp}$. The quadrupole and skew quadrupole corrections had very little effect on the intensity at this operating point, which is consistent with the estimated space charge tune shift.

\section{ACKNOWLEDGMENTS}

The authors would like to thank G. Danby and J. Jackson for their help and advice concerning the skew sextupole

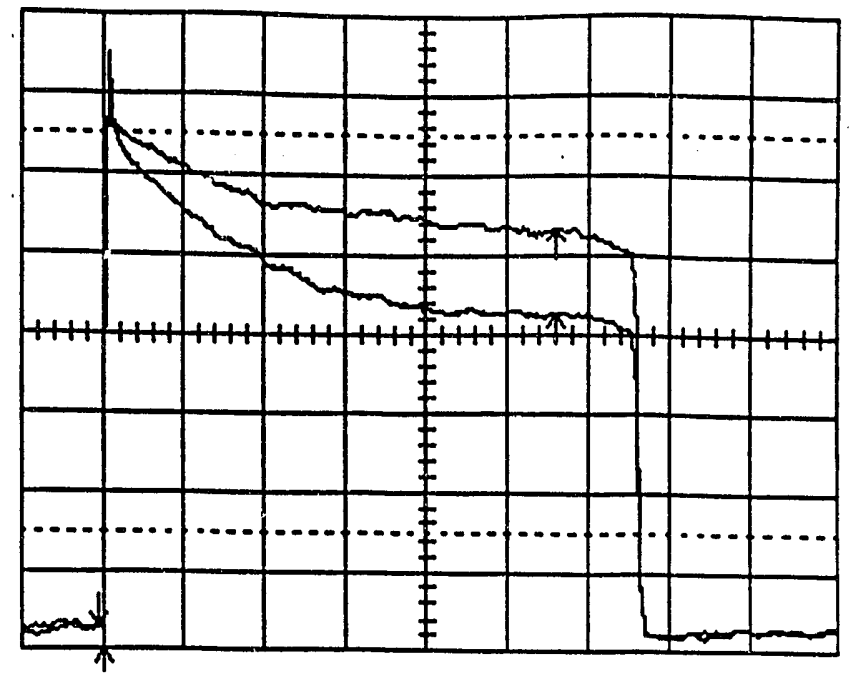

Figure 3: Beam Current with and without Skew Sextupole Correction. Time per division is $10 \mathrm{~ms}$.

corrections and the vacuum chamber self-correction windings. The addition of the skew sextupole and ninth harmonic sextupole corrections would not have been possible without the dedicated and enthusiastic support of the power supply group.

\section{REFERENCES}

[1] L. Ahrens, et al., "The Operational Status of the Booster Injector for the AGS Accelerator Complex at $\mathrm{BNL}^{n}$, XVth Int. Conf. on H.E. Accel., Hamburg, Germany, July 1992, pp. 109-111.

[2] J. Milutinovic, et al., "AGS Booster Orbit and Resonance Correction", Proceedings of the 1989 Particle Accelerator Conference, March 20-23, 1989, pp. 1367-69.

[3] .C. Gardner, "Booster Stopband Corrections", Booster Technical Note No. 217 (unpublished report), AGS Dept., Brookhaven National Laboratory, January 6, 1993.

[4] G. Danby and J. Jackson, "Vacuum Chamber Eddy Current Self-Correction for the AGS Booster Accelerator", Particle Accelerators, 1990, Vol. 27, pp. 33-38. 

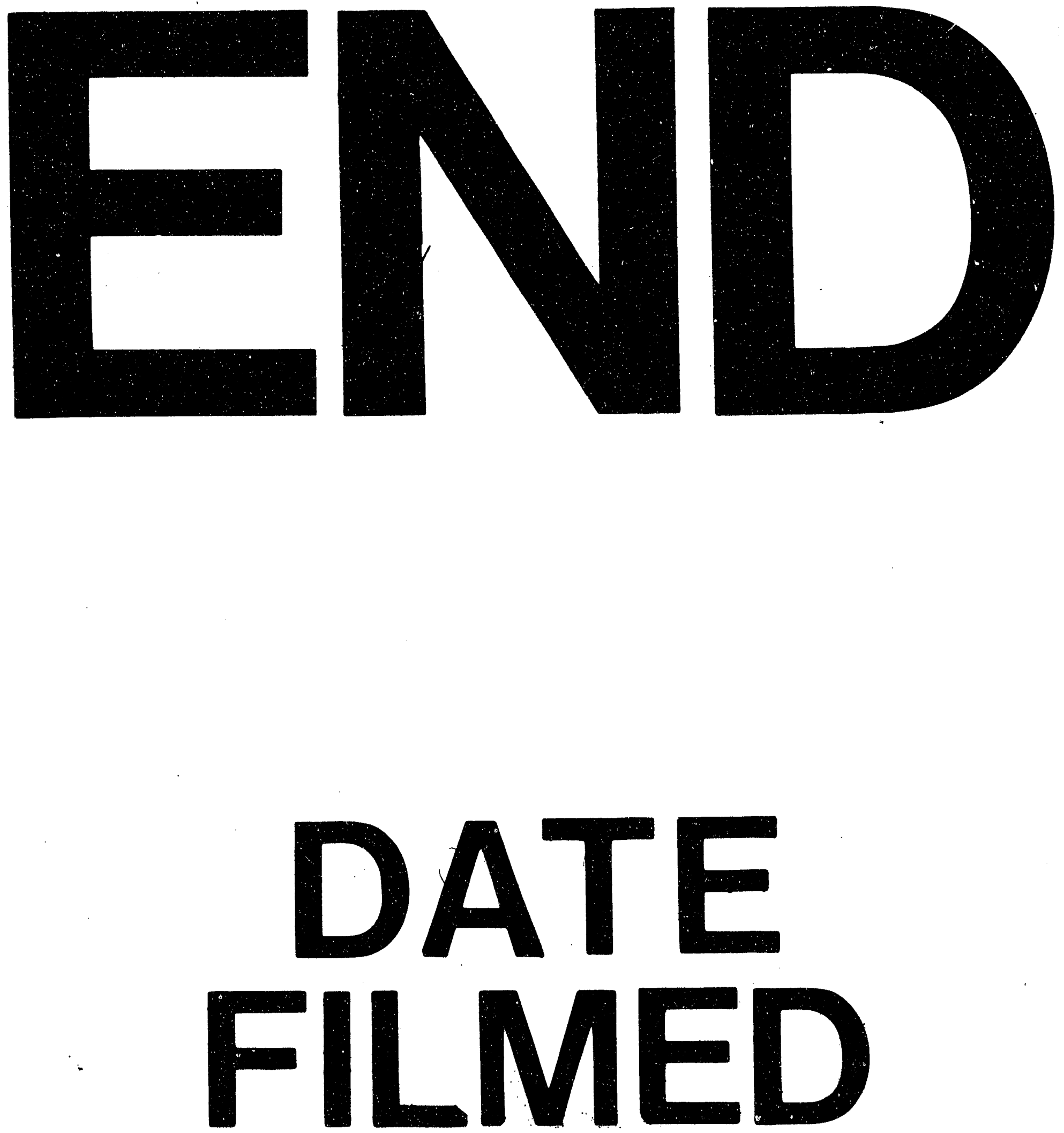

I

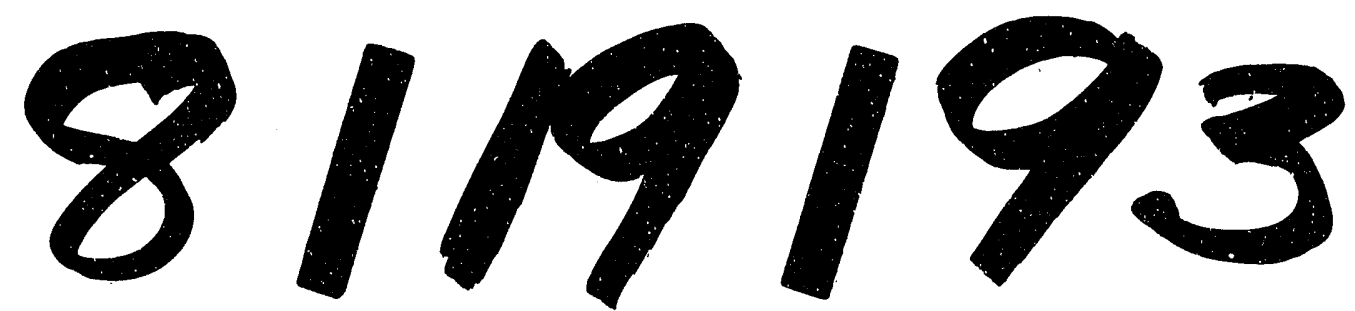


